\title{
Measuring Attitude to School with a Latent Trait Model
}

\author{
Geofferev N. Masters \\ University of Melboupme \\ Norman H. Hyde \\ Education Department of Westem Australia
}

\begin{abstract}
A latent trait model for rating scales is used to analyze responses to an attitude-to-school questionnaire as part of an evaluation of projects operating in 10 Western Australian schools under the Australian Priority Schools Program. The invariance of item parameter estimates over the 10 schools is examined, and variations in item estimates from school to school are studied in the light of the different projects operating in these schools. Results show how the investigation of items that do not retain their difficulties from group to group can provide valuable insight into the ways in which calibration groups differ.

Many recent studies have explored the invariance of latent trait parameter estimates in a variety of testing contexts (e.g., Divgi, 1981; Guskey, 1981; Holmes, 1982; Loyd \& Hoover, 1980; Slinde \& Limn, 1978, 1979). These studies have usually been presented as explorations of the adequacy, or suitability, of particular latent trait models for test equating or as investigations of invariance claims made for these models. The most commonly used model in these studies has been Rasch's (1960/ 1980) dichotomous model.

This paper describes an application of a latent trait model to measure attitude to school across 10 different schools. The model is an extension of Rasch's dichotomous model for rating scales (Andrich, 1978a; Masters, 1980). Although it has been common to approach the invariance question from
\end{abstract}

APPLIED PSYCHOLOGICAL MEASUREMENT Vol. 8, No. 1. Winter 1984, pp. 39-48

(C) Copyright 1984 Applied Psychological Measurement Inc. 0146-6216/84/010039-10\$1.75 the perspective of evaluating the properties of the particular latent trait model being used, this study takes the approach that invariance, or the lack of it, is primarily a feature of the test data. A measurement model cannot guarantee invariant estimates of item difficulty but simply reflects the extent to which items retain their difficulties from group to group. When data fail to produce invariant estimates, the model provides an opportunity to learn something about differences in the definition of the measurement variable for these different groups.

The rating scale model is used here in an exploratory way to uncover differences in the operation of this attitude-to-school variable from school to school. This information was used as feedback to policy makers on the different programs operating in these schools.

\section{Method}

\section{The Questioniliaire}

The Attitude-to-School Questionnaire was developed as part of a federally funded evaluation of programs introduced into 10 Western Australian "priority" schools in 1981. The schools selected are in communities which are believed to contain a high proportion of disadvantaged children. One goal of the programs was to promote children's liking for school. The 25-item questionnaire (see Table 1) was developed to monitor changes in lik- 
Table 1

Attitude to School Questionnaire

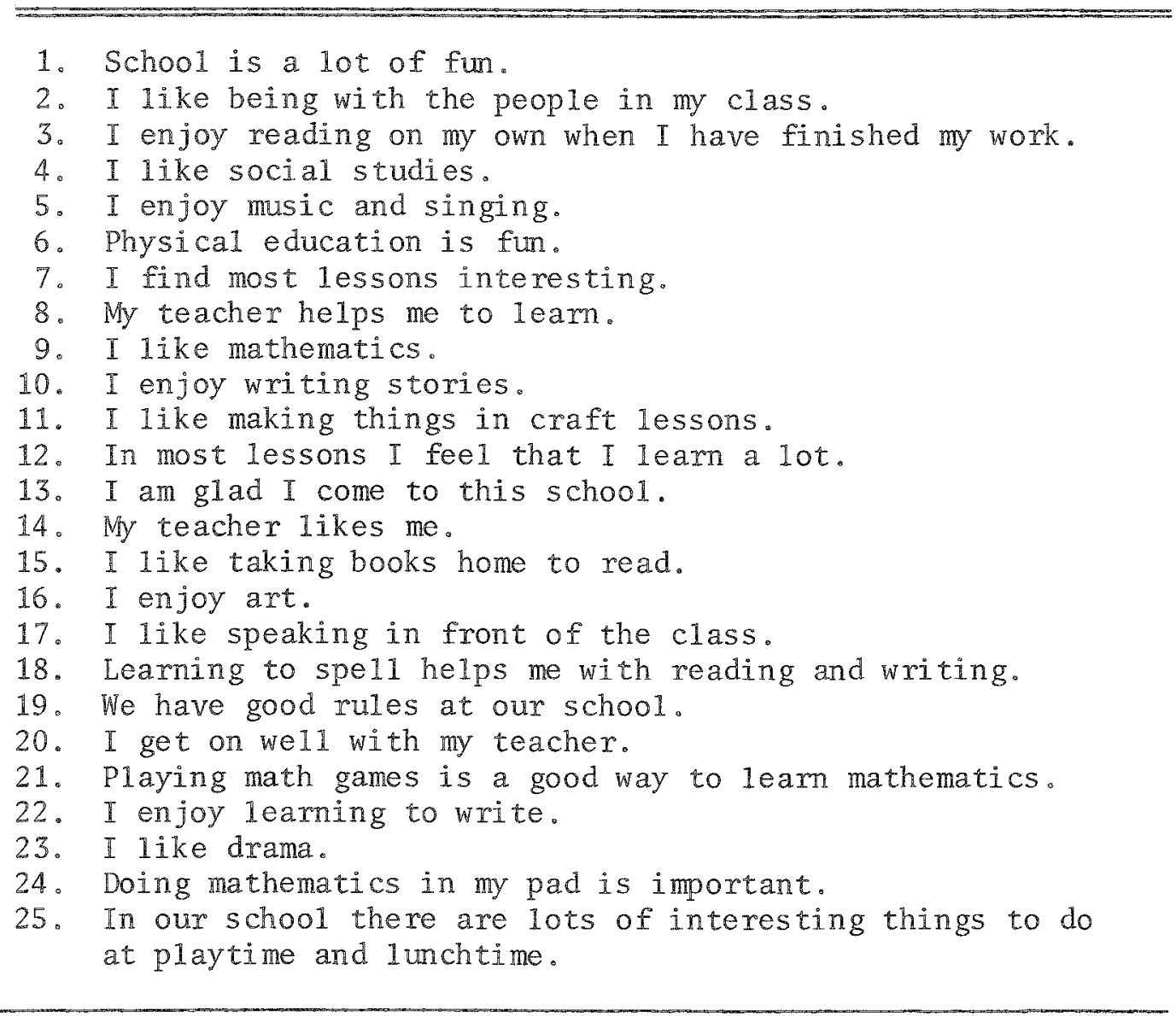

ing for school over time in these 10 priority schools.

The intention of the developers of the Attitudeto-School Questionnaire was to report a single measure of each child's liking for school at any given time. It was anticipated that a positive attitude to school would be reflected in positive statements about (1) school in general (e.g., "School is a lot of fun"); (2) individual school activities (e.g., "I like social studies"); (3) the value of what is done in school (e.g., "Learning to spell helps me with reading and writing'); (4) the student's relationship with his/her teacher (e.g., "I get on well with my teacher'); and (5) the student's relationship with his/her classmates (e.g., "I like being with the people in my class"').

Although these five aspects of a child's attitude to school could be treated as separate subscales and a score reported for each child on each subscale, for the purposes of this analysis, and in keeping with the developers' original intention, the 25 items have been treated as a set. The validity of combining all 25 responses into a single measure of attitude to school has been explored.

Responses to each item on the questionnaire were recorded using the alternatives shown in Figure 1. There has been some discussion in the attitude mea- 
surement literature of possible problems arising from the provision of response alternatives like neutral and undecided (e.g., Andrich, 1978a; Dubois \& Burns, 1975). In particular, there is concem that a noncommittal response altemative may be used as a catchall category by respondents who do not understand an item or who decline to give their opinion, rather than as a position between disagreeing and agreeing. A more serious criticism of neurral response alternatives, however, may stem from their unusual sensitivity to individual differences in response style (Cronbach, 1946). It is common in attitude data to find that some persons with unusual tendencies use only extreme response categories and that others with unusual tendencies give only noncommittal responses. These differences in response style can threaten the validity of attitude measures made with questionnaires that use neutral response alternatives.

An approach that is sometimes taken in an attempt to negate the effects of differences in response style and to improve the fit of attitude data to latent trait models is to recode responses to a smaller number of categories, for example, by combining the undecided and agree responses or the undecided and disagree responses to form only two response categories. However, as Wright and Masters (1982) have demonstrated, an individual's attitude measure can be very different under alterrative but equally plausible recodings of his/her original responses. The recoding of responses to a smaller number of categories may simply hide the effects of measurement disturbances such as differences in personal response style. The approach taken in this study was (1) to analyze responses in the form in which they were collected and (2) to expose differences in response style and to monitor their effects on attitude measurement by testing the fit of individuals to the rating scale model.

\section{Dack Collection and Andysis}

The Attiude-to-School Questionnaire (Table 1) was administered to 876 Year 3 and Year 4 children in 10 Western Australian priority schools in June 1982. Responses to the attitude questionnaire were analyzed with the measurement model for rating
Figgrare 直

The Three Response Altematives

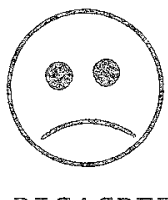

DISAGREE

0
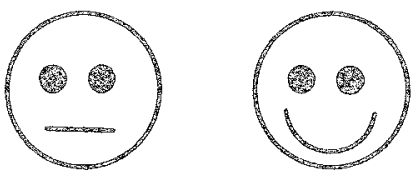

AGREE

2

scales described and applied to a variety of rating scales by Andrich (1978b) and Wright and Masters (1982). The model gives the probability of person $n$ responding in category $x$ to item $i$ as

$$
T^{T_{n i x}}=\frac{\exp \sum_{j=0}^{x}\left(\beta_{n}-\delta_{i}-\tau_{j}\right)}{\sum_{k=0}^{m} \exp \sum_{j=0}^{k}\left(\beta_{n}-\delta_{i}-\tau_{j}\right)}
$$

where

$\beta_{n}$ is the attitude of person $n$,

$\delta_{i}$ is the scalle value of item $i$, and

$\tau_{j}$ governs the probability of a response being made in response category $j$ rather than in category $j-1$.

The 25 items on the attitude questionnaire were calibrated using an extension of $\mathbb{W r i g h t}$ and Panchapakesan's (1969) unconditional maximum likelihood procedure to polytomously scored responses. ${ }^{1}$ As it was not anticipated that the nature of the attitude-to-school variable would vary significantly from school to school, the responses of all 876 children were analyzed jointly in an attempt to define an attitude variable that could be used to compare attitudes across all 10 schools. The 25 items were then calibrated on the responses of children in each school separately, and the stability of the attitude-to-school variable across schools was studied by plotting the various estimates of each item's scale value against each other.

\section{Resdilits}

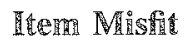

The fit of the attitude-to-school data to the rating scale model was analyzed by calculating the ex-

${ }^{1} \mathrm{~A}$ computer program, CREDIT, for the Rasch analysis of rating scales is available from the MESA Psychometric Laboratory, 5835 S. Kimbark, Chicago IL 60637, U.S.A. 
pected value $E_{n i}$, expected variance $W_{n i}$, and expected kurtosis $C_{n i}$ of each child's response to each item

$E_{n i}=\sum_{k=0}^{m} k P_{n i k}$

$W_{n i}=\sum_{k=0}^{m}\left(k-E_{n i}\right)^{2} P_{n i k}$

$C_{n i}=\sum_{k=0}^{m}\left(k-E_{n i}\right)^{4} P_{n i k}$

where $P_{n i k}$ is child $n$ 's estimated model probability of scoring $k$ on item $i$. When responses to item $i$ fit the rating scale model,

$v_{i}=\sum_{n=1}^{N}\left(x_{n i}-E_{n}\right)^{2} / \sum_{n=1}^{N} W_{n i}$

should be approximately distributed as a mean square with expected value one and expected variance

$q_{i}^{2}=\sum_{n=1}^{N}\left(C_{n i}-W_{n i}^{2}\right) /\left(\sum_{n=1}^{N} W_{n i}\right)^{2}$

The weighted mean square $v_{i}$ can be standardized to

$t_{i}=\left(v_{i}^{1 / 3}-1\right) 3 / q_{i}+q_{i} / 3$

which, when data fit the rating scale model, should have an expected value near zero and an expected standard deviation near one.

Table 2 shows that the item with the largest misfit statistic in this questionnaire is Item 17, "I like speaking in front of the class." Other items which show evidence of poor fit to the model are litem 14, "My teacher likes me", and Item 22, "I enjoy learning to write." To explore the nature of the misfit of these three items, the 795 children who scored between $\mathbb{1}$ and 49 on the attitude-to-school questionnaire were divided into four attitude groups corresponding to questionnaire scores of $1-34,35-$ $39,40-44$, and 45-49. The proportions of children in each of these attitude groups responding disagree, undecided, and agree to Items 17, 14, and 22 are shown in Figure 2.

The smooth curves in Figure 2 are the estimated category probability curves for these three items. They show the ways in which the model probabilities of responding disagree (d), undecided (u) and agree (a) to each of these items vary with liking for school. Superimposed over the model proba- bility curves are the observed proportions of children in each attitude group making each response. By comparing the observed proportions and model probabilities, the nature of each item's misfit can be studied.

The plot for the most poorly fitting item, Item 17 , shows that for the group of children with the most positive attitudes, on the right of Figure 2, fewer children than expected agree and more children than expected are undecided or disagree. In other words, fewer high scoring children than expected enjoy "speaking in front of the class."

For the group of children on the left of Figure $2 \mathrm{a}$, who like school least, fewer children than expected disagree with Item 17, and more children than expected are undecided or agree. This pattern of observed proportions is typical of an item that is not highly correlated with the other questionnaire items. Item 17 does not work with the majority of these items to define a coherent variable of increasing liking for school and so should probably be deleted from the questionnaire.

Item 14, "My teacher likes me," is also poorly fitting (Figure 2b). The striking feature for this item is the surprising number of children at all attitude levels who responded undecided. The misfit of Item 14 might stem from its invitation to children to guess at how their teacher feels about them. The children are reluctant to guess their teachers' feelings. This phenomenon does not occur for Item 20 , "I get on well with my teacher," where the proportions of children responding undecided were very similar to the estimated probabilities.

The model probabilities and observed proportions for the third most poorly fitting item, Item 22 , "I enjoy learning to write," are shown in Figure $2 \mathrm{c}$. For this item the observed proportions are much closer to the model probabilities. If there is a problem with this item, it appears to be that it is too discriminating, with more children than expected in the higher attitude groups agreeing and more children than expected in the lowest attitude group disagreeing. However, the misfit of Item 22 may not be sufficient to warrant its removal from this questionnaire. The other 22 items all have fit statistics closer to their expected value than do Items 17,14 , and 22 (see Table 2). 
Table 2

Attitude to School Item Statistics

\begin{tabular}{|c|c|c|c|c|c|}
\hline Item & $\begin{array}{c}\text { Scale value } \\
\mathrm{d}_{\mathbf{i}} \\
\end{array}$ & $\begin{array}{c}\text { Error } \\
\mathrm{s}_{\mathrm{i}} \\
\end{array}$ & $\begin{array}{c}\text { Mean Square } \\
v_{i} \\
\end{array}$ & $\begin{array}{c}\text { Error } \\
\mathrm{q}_{\mathrm{i}} \\
\end{array}$ & $\begin{array}{r}\text { Fi t } \\
t_{i} \\
\end{array}$ \\
\hline 17 & 1.59 & .05 & 1.18 & .05 & 3.53 \\
\hline 14 & .55 & .05 & .83 & .05 & -3.42 \\
\hline 9 & .48 & .05 & .97 & .05 & -.59 \\
\hline 7 & .36 & .05 & 1.03 & .04 & .64 \\
\hline 4 & .35 & .05 & .99 & .05 & -.23 \\
\hline 20 & .34 & .05 & .99 & .05 & -.17 \\
\hline 3 & .29 & .06 & .91 & .06 & -1.60 \\
\hline 15 & .25 & .06 & 1.08 & .06 & 1.36 \\
\hline 10 & .20 & .06 & .92 & .05 & -1.55 \\
\hline 23 & .19 & .06 & .95 & .06 & -.83 \\
\hline 1 & .15 & .06 & 1.02 & .05 & .51 \\
\hline 24 & .11 & .06 & .95 & .06 & -.82 \\
\hline 12 & .11 & .06 & .98 & .06 & -.27 \\
\hline 19 & .09 & .06 & .89 & .06 & -1.91 \\
\hline 25 & .06 & .06 & 1.01 & .05 & .31 \\
\hline 13 & .04 & .06 & 1.10 & .05 & 2.01 \\
\hline 5 & -.05 & .06 & 1.01 & .05 & .14 \\
\hline 22 & $=.18$ & .06 & 1.15 & .06 & 2.40 \\
\hline 2 & -.20 & .06 & .93 & .06 & -1.12 \\
\hline 6 & -.31 & .06 & 1.08 & .05 & 1.61 \\
\hline 21 & -.32 & .06 & 1.03 & .05 & .68 \\
\hline 18 & -.52 & .07 & 1.14 & .07 & 1.92 \\
\hline 11 & -.99 & .08 & $\mathbb{1} .00$ & .07 & .03 \\
\hline 8 & -1.12 & .09 & 1.02 & .08 & .30 \\
\hline 16 & -1.48 & .11 & .85 & .09 & -1.72 \\
\hline Mean & .00 & .06 & 1.00 & .06 & .05 \\
\hline S.D. & .60 & .01 & .09 & .01 & 1.55 \\
\hline
\end{tabular}

\section{Factor Analysis}

As a further check on the validity of combining responses to the 25 attitude-to-school items and reporting a single score for each child, a factor analysis of these data was conducted. Of particular concern was the possibility that a significant, and theoretically interesting, second dimension may have been overlooked among the items with moderate levels of misfit. Results of the analysis show that the first factor (eigenvalue $=6.35$ ) accounted for
$25.5 \%$ of the variance, while the second factor (eigenvalue $=1.37$ ) accounted for only $5.5 \%$ of the variance. This supports the conclusion of the latent trait analysis that these attitude-to-school data are essentially unidimensional.

Rotated factor loadings for the first two factors are shown in Table 3. The second factor is defined by Items 11 , "I like making things in craft lessons," and Item 16, "I enjoy art." These items have very low scale values and may simply define 
Fingure 2

Model Probabilities and Observed Proportions for the Three Poorest Fitting Items

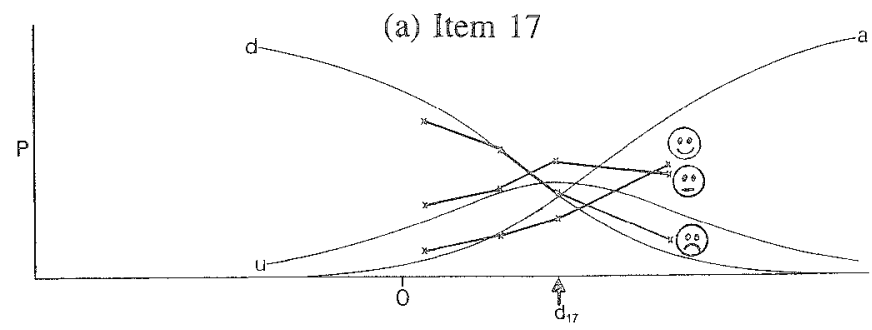

(b) Item 14

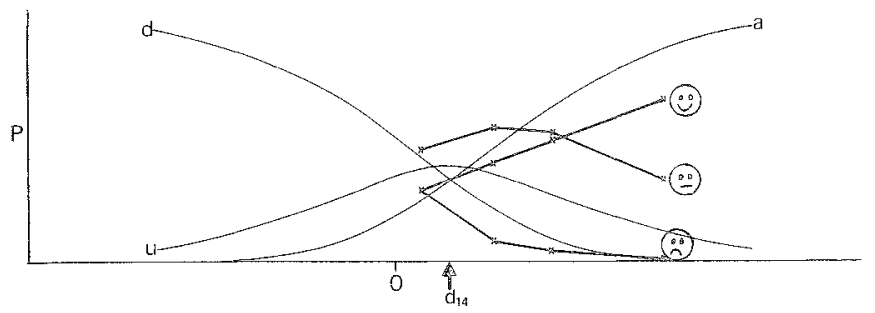

(c) Item 22

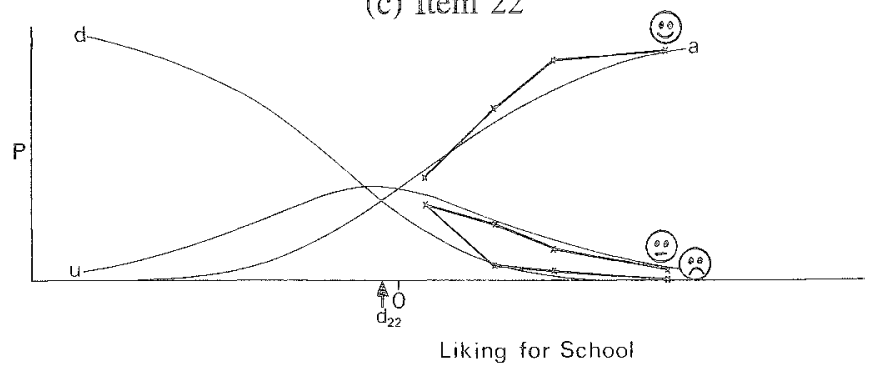

a difficulty factor. In any case, there is no evidence of a significant second dimension in these data.

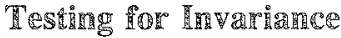

To study the invariance of the item scale value estimates over the 10 schools, the questionnaire items were also calibrated on each school separately, and the resulting scale value estimates compared with the estimates based on the responses of all 795 children. Results for two of the 10 schools are shown in Figures 3 and 4.

Figure 3 shows the scale value estimates for School 4. This particular plot is considered here because of the relatively high correlation between the School 4 estimates and the estimates based on all 10 schools. (Only School 10 had estimates that were more con- sistent with the all schools estimates). All but one of the 25 questionnaire items in Figure 3 are inside the $95 \%$ confidence interval. (The procedure for constructing this interval is described by Wright \& Masters, 1982, p. 115).

Item 6, "Physical education is fun," had a significantly lower scale value in School 4 than in the other nine schools. In other words, children in School 4 found it unusually easy to agree with Item 6 . When it is calibrated on the responses of all 795 children, physical education is estimated to be only slightly more enjoyable than "leaming to write" (Item 22). However, for the 85 children in School 4 , physical education is estimated to be much more enjoyable than learning to write and more enjoyable even than "crafts" (Item 11). Children in School 4 found it easier to agree that physical ed- 
Table 3

Factor Analysis Results

\begin{tabular}{|c|c|c|}
\hline Item & $\begin{array}{c}\text { Factor } \\
1 \\
\end{array}$ & $\begin{array}{c}\text { Factor } \\
2 \\
\end{array}$ \\
\hline 1 & .61 & .08 \\
\hline 2 & .41 & .14 \\
\hline 3 & .39 & .20 \\
\hline 4 & .50 & .20 \\
\hline 5 & .32 & .31 \\
\hline 6 & .33 & .07 \\
\hline 7 & .51 & .31 \\
\hline 8 & .36 & .19 \\
\hline 9 & .53 & .14 \\
\hline 10 & .37 & .21 \\
\hline 11 & .00 & .77 \\
\hline 12 & .57 & .26 \\
\hline 13 & .64 & .11 \\
\hline 14 & .53 & -.04 \\
\hline 15 & .46 & .31 \\
\hline 16 & .02 & .81 \\
\hline 17 & .45 & .12 \\
\hline 18 & .50 & .27 \\
\hline 19 & .57 & .16 \\
\hline 20 & .65 & .04 \\
\hline 21 & .36 & .40 \\
\hline 22 & .51 & .28 \\
\hline 23 & .25 & .40 \\
\hline 24 & .52 & .29 \\
\hline 25 & .37 & .18 \\
\hline
\end{tabular}

ucation is fun than to concede that learning to spell is useful (Item 18) or that mathematics games are a good way to learn mathematics (Item 21).

According to School 4's project proposal, no special initiatives in physical education were planned by this school. Thus, there is no obvious explanation for the lower scale value of Item 6 in terms of this school's proposed program. This is not to say that the unusually high level of agreement with this item cannot be traced to the physical education activities actually being provided in School 4. If some special feature of these activities can be identified as contributing to unusual enjoyment of phys- ical education, then this may provide a useful model for teachers in other schools.

To explore the low scale value of Item 6 in School 4 further, the expected and observed frequencies of children responding disagree, undecided, and agree to Item 6 were calculated and are displayed in Table 4. The 85 children were divided into four attitude groups, with children in Group $\mathbb{1}$ having the least positive attitudes and children in Group 4 having the most positive attitudes to school. The model frequencies in Table 4 are based on the attitude estimates and item scale value estimate from the analysis of all 795 response records.

Table 4 shows that fewer School 4 children than expected responded disagree or undecided to Item 6. Among children with the least positive attitudes to school (Groups $\mathbb{1}$ and 2), more children than expected responded agree. It was their unexpected agreement that produced the surprisingly low estimate for this item. The observation that the low scale value of Item 6 in School 4 is accounted for by the unexpected agreement of only six or seven of these 85 children raises the possibility that it has occurred by chance, rather than as a result of something peculiar to School 4.

Figure 4 shows the scale value estimates for School 6 , the school in which the 25 estimates were least consistent with the all schools estimates. Four of the questionnaire items are outside the $95 \%$ confidence interval. Children in School 6 found it unusually easy to agree with Item 2, "I like being with the people in my class," Item 7, "I find most lessons interesting," and Item 4, "I like social studies," and unusually difficult to agree with Item 25, "In our school there are lots of interesting things to do at playtime and lunchtime."

The unexpectedly high scale value of Item 25 in School 6 is not surprising in view of that school's physical setting: School 6 has the poorest physical facilities of these 10 schools. There is no grassed playing area and no covered area. The school yard contains areas of black sand, and there is little for children to do during playtime and lunchtime.

An alternative approach to analyzing the stability of the liking for school variable over these 10 schools is taken in Figure 5. The 10 scale value estimates 
Figure 3

Item Scale Values for School 4 vs. All Schools

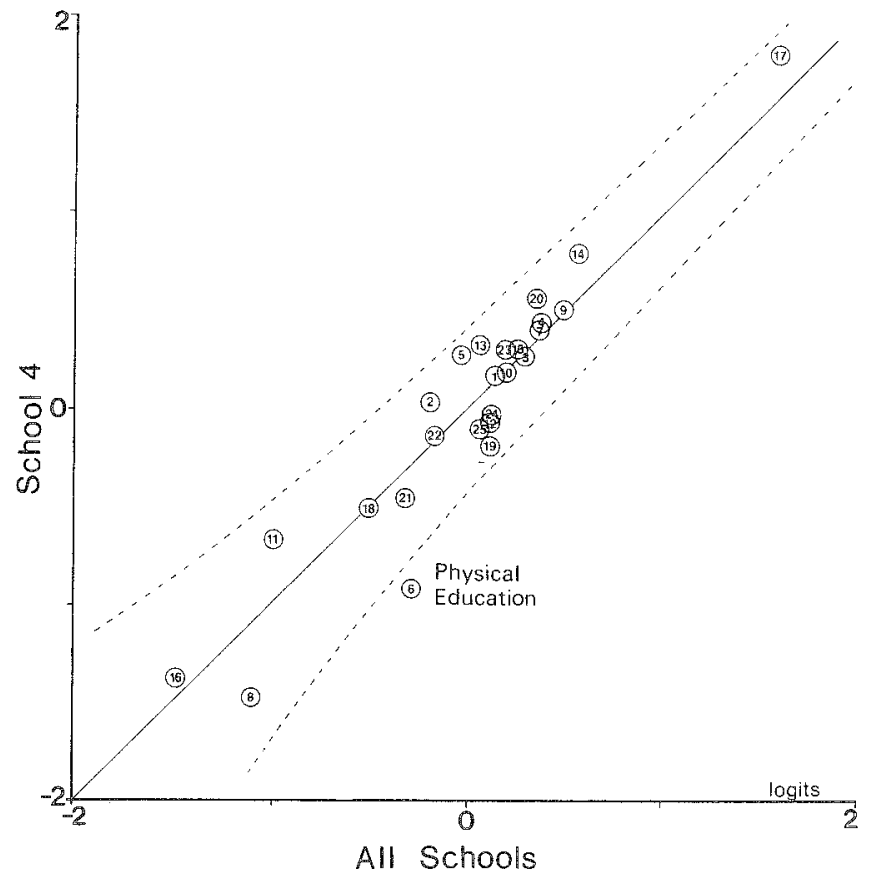

Figure 4

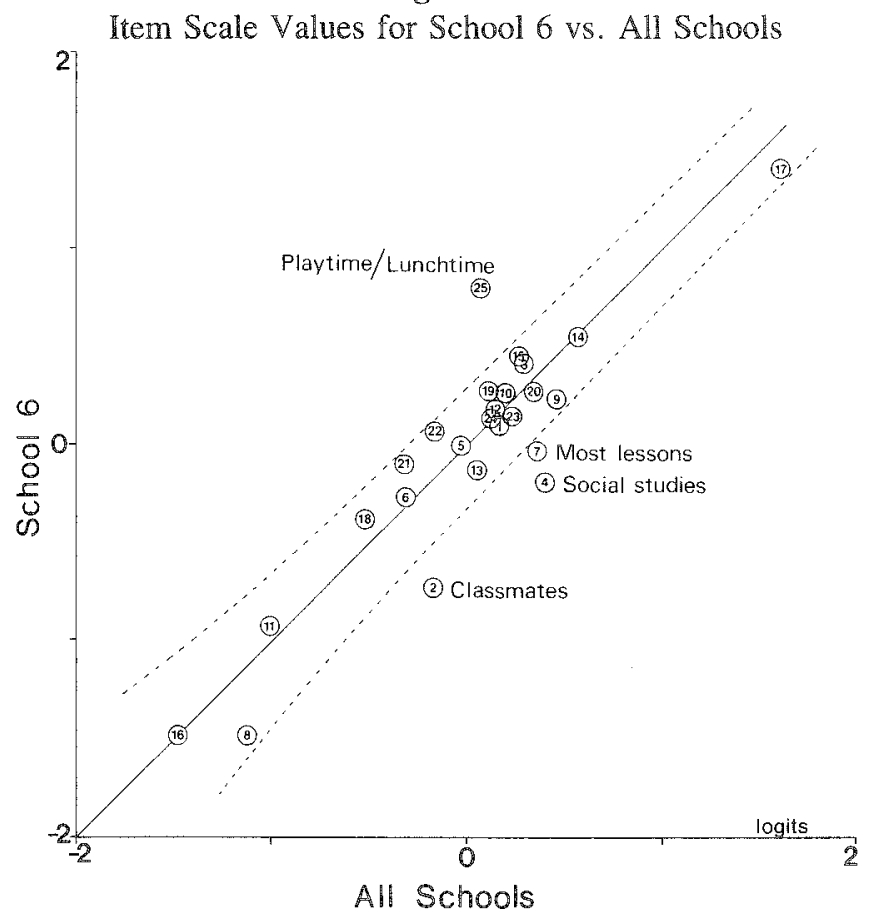

Downloaded from the Digital Conservancy at the University of Minnesota, http://purl.umn.edu/93227. May be reproduced with no cost by students and faculty for academic use. Non-academic reproduction requires payment of royalties through the Copyright Clearance Center, http://www.copyright.com/ 
Table 4

Model and Observed Frequencies of Response: School 4, Irem 6

\begin{tabular}{lrrr}
\hline \hline & Model & Obsvd & Diff \\
\hline Group 1 & & & \\
Disagree & 2.5 & 1 & -1.5 \\
Undecided & 6.9 & 5 & -1.9 \\
Agree & 13.6 & 17 & $\mathbf{3 . 4}$ \\
Group 2 & & & \\
Disagree & 1.1 & 0 & -1.1 \\
Undecided & 5.1 & 3 & -2.1 \\
Agree & 15.8 & 19 & $\mathbf{3 . 2}$ \\
Group 3 & & & \\
Disagree & 0.5 & 0 & -0.5 \\
Undecided & 3.5 & 4 & 0.5 \\
Agree & 21.0 & 21 & 0.0 \\
Group 4 & & & \\
Disagree & 0.0 & 0 & 0.0 \\
Undecided & 0.6 & 0 & -0.6 \\
Agree & 14.4 & 15 & 0.6 \\
& & & \\
\hline
\end{tabular}

for Items 17,13 , and 25 are plotted on the same line and compared with the all schools estimates (arrowed); a confidence interval corresponding to two standard errors of estimate is shown about each estimate. The 10 estimates for Item 13 , in the middle of Figure 5, are closely grouped about the all schools estimate. Item 13 is estimated to have much the same scale value in all 10 schools.

The estimates for Item 25 are more widely dispersed about the all schools estimate. As noted earlier, children in School 6 found it unusually difficult to agree with Item 25 . Their responses have made the all schools estimate for Item 25 higher than it would otherwise have been.

Item 17 is not only the item most difficult to agree with but is also the most poorly fitting item in this questionnaire. Figure 5 shows that it has a high scale value in all schools except School $\mathbb{1}$. The unusual behavior of Item 17 in School 1 has probably contributed to this item's misfit.

School 1 is in a particularly disadvantaged area. Most children live in high density public housing, and an unusually high proportion belong to single parent families. As part of the program introduced into this school, a special effort was made to promote self-confidence, self-esteem, and cooperation

Fingergre 5

Scale Value Estimates from All 10 Schools for Items 17, 13, and 25

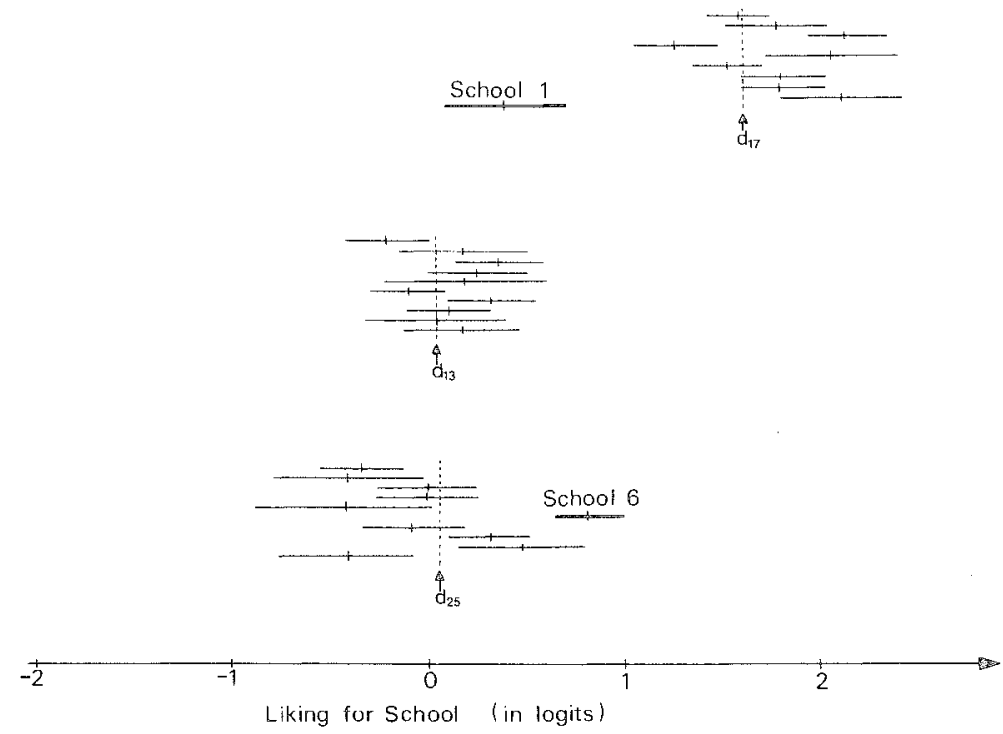

Downloaded from the Digital Conservancy at the University of Minnesota, http://purl.umn.edu/93227. May be reproduced with no cost by students and faculty for academic use. Non-academic reproduction requires payment of royalties through the Copyright Clearance Center, http://www.copyright.com/ 
among children. Whether or not this has contributed to the unusually positive responses of children in School 1 to Item 17 cannot be ascertained from these data. However, if the low scale value of Item 17 in School 1 has resulted from some special initiative being taken in this school, that would certainly warrant closer investigation.

\section{DîSCUESSion}

Although a number of latent trait models for ordered response category data have been proposed, as yet there have been few applications of these models to practical measurement problems. The attitude-to-school data analyzed here appear to fit the rating scale model quite well. At least two questionnaire items function somewhat differently from the others. Responses to these two items should probably not be combined with responses to the other 23 items.

The study of items which have significantly lower or significantly higher scale value estimates in some schools has drawn attention to special features of particular schools. An unusually high level of agreement with an item in a particular school may help identify some special initiative being taken in that school which could be used as a model by other schools. An unusually low level of agreement with an item may help identify a need for special assistance or intervention. Although most studies of invariance have been presented as explorations of invariance claims made for latent trait models, in this study variations in item estimates have been used to expose and to explore differences among calibration groups. Results suggest that this use of latent trait theory may be of considerable value to measurement practitioners.

\section{Referentes}

Andrich, D. A binomial latent trait model for the study of Likert-style attitude questionnaires. British Journal of Mathematical and Statistical Psychology, 1978, 31 , 84-98. (a)
Andrich, D. Application of a psychometric rating model to ordered categories which are scored with successive integers. Applied Psychological Measurement, 1978, 2, 581-594. (b)

Cronbach, L. J. Response sets and test validity. Educational and Psychological Measurement, 1946, 6, 475-494.

Divgi, D. R. Model-free evaluation of equating and scaling. Applied Psychological Measurement, 1981, 5, 203-208

Dubois, B., \& Burns, J. A. An analysis of the meaning of the question mark response category in attitude scales. Educational and Psychological Measurement, $1975,35,869-884$.

Guskey, T. R. Comparison of a Rasch model scale and the grade-equivalent scale for vertical equating of test scores. Applied Psychological Measurement, 1981, 5, 187-201.

Holmes, S. E. Unidimensionality and vertical equating with the Rasch model. Journal of Educational Measurement, 1982, 19, 139-147.

Loyd, B. H., \& Hoover, H. D. Vertical equating using the Rasch model. Journal of Educational Measurement, 1980, 17, 179-193.

Masters, G. N. A Rasch model for rating scales. Unpublished doctoral dissertation, University of Chicago, 1980.

Rasch, G. Probabilistic models for some intelligence and attainment tests. Copenhagen, Denmark: Danmarks Paedogigiske Institut, 1960 (Chicago: University of Chicago Press, 1980).

Slinde, J. A., \& Linn, R. L. An exploration of the adequacy of the Rasch model for the problem of vertical equating. Journal of Educational Measurement, 1978, 15, 23-35.

Slinde, J. A., \& Lim, R. L. The Rasch model, objective measurement, equating and robustness. Applied Psychological Measurement, 1979, 3, 437-452.

Thurstone, L. L. Attitudes can be measured. American Journal of Sociology, 1928, 33, 529-554.

Wright, B. D., \& Masters, G. N. Rating scale analysis. Chicago: MESA Press, 1982.

Wright, B. D., \& Panchapakesan, N. A procedure for sample-free item analysis. Educational and $P_{\text {sycho- }}$ logical Measurement, 1969, 29, 23-48.

\section{Author's Address}

Send requests for reprints or further information to Geofferey N. Masters, Centre for the Study of Higher Education, University of Melbourne, Parkville, Victoria, Australia, 3052. 